\title{
Desenvolvimento motor e sua influência em testes de leitura e psicogênese de escolares
}

\author{
Shirley Holanda da Silva Rocha ${ }^{1}$ José Fernando Vila Nova de Moraes ${ }^{2}$ Isabela Almeida Ramos ${ }^{3}$ Suliane Beatriz Rauber ${ }^{4}$ \\ Lílian Alves Pereira ${ }^{3}$ Herbert Gustavo Simões ${ }^{5}$ Carmen Sílvia Grubert Campbell ${ }^{6}$
}

\begin{abstract}
RESUMO
O desenvolvimento motor e o cognitivo estão relacionados, com isso, o prejuízo em um deles pode influenciar a evolução do outro. Sendo assim, o objetivo do presente estudo foi investigar a influência do desenvolvimento motor no desempenho da leitura e escrita em escolares de 7 a 9 anos. Foi realizado um estudo transversal com 30 crianças de ambos os sexos, estudantes de uma escola da rede distrital de ensino da cidade de Ceilândia - DF. Foram aplicados testes motores e testes de leitura e psicogênese, e foram mensurados a massa corporal, estatura, índice de massa corporal e pressão arterial. Por meio do Teste Exato de Fisher, foram observadas associações entre o nível de psicogênese e a escala de desenvolvimento motor, idade motora geral e quociente motor geral. A Correlação de Spearman associou significativamente o nível da psicogênese e a escala de desenvolvimento motor, idade motora geral e quociente motor geral. A Correlação de Spearman também apontou associações significativas entre teste de leitura, a idade motora geral e o quociente motor geral; entretanto, as variáveis antropométricas não se associaram aos índices motores ou cognitivos. Com isso, observa-se que houve associação entre os aspectos motores e cognitivos nas crianças estudadas.
\end{abstract}

Descritores: Crianças; Desenvolvimento Motor; Desenvolvimento Cognitivo; Aprendizagem.

\section{Motor development and its influence in students' reading and pshycogenesis tests}

\begin{abstract}
Motor and cognitive developments are associated and an impairment in the evolution of one of them can influence the other's progression. Therefore, the aim of the present study was to investigate the influence of motor development in reading and psychogenesis of 7 to 9 year old children. In order to do so, a cross-sectional study involving 30 children of both sexes, students of a public school in the city of Ceilândia - DF took place. They performed motor tests and reading and psychogenesis tests. Body mass, stature, body mass index and blood pressure were also measured. Fisher's Exact Test showed significant associations between the level of psychogenesis and the motor development scale, global motor age and global motor quotient. Spearman's Correlation revealed significant associations between the level of psychogenesis and the motor development scale, global motor age and global motor quotient. Moreover, Spearman's Correlation also showed significant correlations between the reading test and global motor age, and global motor quotient. However, no associations were found when comparing the anthropometric variables and the motor and cognitive indexes. Hence, it is concluded that there was an association between the motor and cognitive aspects in studied children.
\end{abstract}

Descriptors: Children; Motor Development; Cognitive Development; Learning.

\footnotetext{
${ }^{1}$ Graduada em Educação física pela Universidade Católica de Brasília (UCB), Brasília, DF, Brasil.

${ }^{2}$ Doutor em Educação física pela Universidade Católica de Brasília (UCB), Brasília, DF, Brasil.

${ }^{3}$ Mestre em Educação física pela Universidade Católica de Brasília (UCB), Brasília, DF, Brasil.

${ }^{4}$ Mestranda em Educação física na Universidade Católica de Brasília (UCB), Brasília, DF, Brasil.

${ }^{5}$ Pós doutorado em Educação Física pela Universidade Federal de Juiz de Fora (UFJF), Juiz de Fora, MG, Brasil.

${ }^{6}$ Doutor em Ciências pela Universidade Federal de São Paulo (USP), São Paulo, SP, Brasil.
} 


\section{Introdução}

Os anos iniciais da vida de uma criança envolvem etapas de evolução do seu desenvolvimento de forma gradativa e complexa, constituindo um processo de mudanças. Nessa etapa, a criança desenvolve as áreas afetiva, cognitiva, social e motora em plenitude, o que proporciona maior domínio dos diversos movimentos, raciocínios e interações sócioafetivas com o meio em que vive ${ }^{1}$. Estas dimensões estão integradas e inter-relacionadas, gerando um desenvolvimento sequencial. No entanto, o ritmo e o tempo que determinam esse processo variam de acordo com a cultura e a individualidade da criança ${ }^{2}$. Assim, quanto mais integração houver entre essas dimensões, mais global e facilitada será a aprendizagem da criança.

Segundo Newcombe ${ }^{3}$, o desenvolvimento envolve mudanças que acontecem de forma ordenada e influenciam neurologicamente e fisicamente todos os comportamentos pertinentes ao ser humano. O desenvolvimento infantil é contínuo e, por meio dele, a criança acumula capacidades cada vez mais complexas de se locomover, pensar, interagir, sendo um processo duradouro, longo e iniciado desde quando somos gerados3.

No que tange à psicogênese da língua escrita, Ferreiro e Teberosky 4 identificaram estágios progressivos ao longo da aquisição da escrita, partindo do níveis pré-silábico até o alfabetizado. Esses compõem uma série de modos de representação que se desenvolvem até o nível da representação alfabética da linguagem. De acordo com Le Boulch ${ }^{5}, 0$ domínio da língua escrita é submetido a um conjunto de condições que envolvem o domínio da linguagem, a familiarização global com código gráfico e condições psicomotoras.

Toda aprendizagem ocorre por meio do corpo, onde os órgãos sensoriais e os membros são portas de comunicação, com entrada e saída de informações processadas pelo Sistema Nervoso Central (SNC). Quem escreve é um sujeitocriança, porém, para fazê-lo, necessita de sua mão, de sua orientação espacial (lateralidade), de um ritmo motor (relaxamento-contração), de sua postura (eixo postural), de sua tonicidade muscular (preensão fina e precisa) e de seu reconhecimento no referido ato (função imaginária) ${ }^{6}$. Dessa forma, a educação psicomotora ajuda as crianças a desenvolverem sua unidade e globalidade de ser humano, sua organização espacial, temporal e sua relação afetiva por meio das interações ${ }^{7}$.

Em relação à cognição, estudos apontam que diferentes tipos de atividade física podem ter uma influência favorável sobre várias medidas de desempenho cognitivo em crianças, como por exemplo, o quociente de inteligência (QI), a realização dos testes verbais e de matemática ${ }^{7}$, bem como a capacidade de memorização e concentração ${ }^{8}$.

Nesse sentido, o desenvolvimento motor e o cognitivo estão relacionados. Visto que, frequentemente, quando há perturbações genéticas ou ambientais que afetam a coordenação motora ou cognitiva, usualmente observam-se prejuízos nas duas partes, ao invés de apenas uma ${ }^{7}$. Com isso, o objetivo do presente estudo foi investigar a influência do desenvolvimento motor no desempenho da leitura e escrita em escolares de 7 a 9 anos.

\section{Metodologia}

\section{Amostra, aspectos éticos e delineamento}

O presente estudo foi realizado entre abril e junho de 2010, na cidade de Ceilândia - DF. A amostra foi composta por 30 crianças, de ambos os sexos e idade entre 7 e 9 anos, estudantes de uma turma da terceira etapa de alfabetização de uma escola da rede distrital de ensino. $O$ delineamento foi transversal e possibilitou avaliar a relação entre o desenvolvimento motor e o desempenho em testes de leitura e escrita dos escolares.

Após aprovação pelo Comitê de Ética e Pesquisa (CEP), da Universidade Católica de Brasília (UCB), protocolo $n^{0}$. 077/2010, os pais assinaram um termo de consentimento livre e esclarecido autorizando seus filhos a participarem do estudo. Como critérios de inclusão do escolar na amostra este deveria ter autorização prévia do responsável, não ser repetente, não apresentar deficiência física, dificuldade de aprendizagem, déficit sensorial e alterações comportamentais ou mentais. Todos os testes foram aplicados por um avaliador experiente com formação em pedagogia e educação física.

\section{Testes motores}

A avaliação motora das crianças foi pela bateria de testes motores, propostos por Rosa Neto ${ }^{9}$. Os testes foram aplicados de acordo com a idade cronológica das crianças, organizados progressivamente em grau de complexidade, sendo atribuído para cada tarefa, em caso de êxito, um valor correspondente à idade motora (IM), expressa em meses. 
Brevemente, os testes englobam os seguintes componentes da motricidade: motricidade fina: enfiar uma linha na agulha, fazer um nó, percorrer um labirinto usando lápis e papel, fazer bolinhas de papel com uma das mãos, tocar os demais dedos da mesma mão com a ponta do polegar e lançar uma bola; motricidade global: saltar sobre o mesmo lugar, saltar uma altura de $20 \mathrm{~cm}$, caminhar em linha reta, andar com o apoio de apenas um pé, saltar uma altura de $40 \mathrm{~cm}$ e saltar e tocar os calcanhares com as mãos; por fim, equilíbrio: na ponta dos pés, de cócoras, com o tronco flexionado, fazer uma forma de quatro, ficar na ponta dos pés com os olhos fechados e andar com o apoio de apenas um pé com os olhos fechados.

A partir dos resultados obtidos, foi realizada uma classificação que considerava a escala de desenvolvimento motor, a idade motora geral e o quociente motor geral (idade motora geral : idade cronológica).

\section{Teste cognitivo}

Os testes cognitivos, segundo protocolo de Ferreiro ${ }^{9}$, consistem em o avaliador contar uma história ou cantar música para posteriormente retirar dessa fonte quatro palavras, sendo: uma monossílaba, uma dissílaba, uma trissílaba, uma polissílaba e uma frase. As palavras foram ditadas aos alunos que escreveram de acordo com o nível de desenvolvimento da escrita, como demonstrado na Figura 1.

A partir dos resultados, foi analisado o nível da psicogênese do escolar e classificados nos seguintes níveis: présilábico I e II, silábico, silábico-alfabético, alfabético e alfabetizado I, II, III e IV¹0.

\begin{tabular}{|c|c|}
\hline $\begin{array}{l}\text { EEEE } \\
\text { (CÃO) }\end{array}$ & \begin{tabular}{|r}
$2-$ \\
\\
UU \\
\\
(URSO)
\end{tabular} \\
\hline $\begin{array}{c}\text { ACAE } \\
\text { (JACARÉ) }\end{array}$ & $\begin{array}{c}\text { AAIO } \\
\text { (PASSARINHO) }\end{array}$ \\
\hline $\begin{array}{l}\text { 1- O CAIAOIO } \\
\text { (O PASSARINHO AL }\end{array}$ & S BICHOS). \\
\hline
\end{tabular}

Figura 1 - Teste de um indivíduo com nível de escrita silábica.

\section{Medidas antropométricas e pressão arterial}

Para caracterização da amostra, foram mensuradas a massa corporal em uma balança eletrônica (Tech 05, China) e a estatura (estadiômetro de parede), a fim de obter o cálculo do índice de massa corporal (IMC). A pressão arterial foi aferida pelo método auscultatório, com o voluntário sentado, e foi utilizando esfigmomanômetro pediátrico da marca BD, de manguito adequado à circunferência do braço da criança e estetoscópio infantil (Missouri), por uma única avaliadora, com a criança em repouso, por no mínimo 5 minutos.

\section{Análise estatística}

Foram realizados o Teste Exato de Fisher e a Correlação de Spearman para verificar a associação entre as variáveis motoras, cognitivas e antropométricas. 0 nível de significância adotado foi $p \leq 0,05$ e o programa utilizado para a análise dos dados foi o SPSS 15.0 (SPSS Inc., Estados Unidos). 


\section{Resultados e discussão}

As características gerais dos participantes podem ser encontradas na tabela 1.

Tabela 1 - Característica geral da amostra $(n=30)$. Dados expressos em média \pm desvio-padrão.

\begin{tabular}{c|c}
\hline Idade (anos) & $8,2 \pm 0,5$ \\
\hline Massa Corporal $(\mathrm{kg})$ & $30,0 \pm 6,8$ \\
\hline Estatura $(\mathrm{cm})$ & $128,3 \pm 6,1$ \\
\hline Índice de massa corporal $\left(\mathrm{kg} / \mathrm{m}^{2}\right)$ & $18,0 \pm 3,6$ \\
\hline Pressão arterial sistólica $(\mathrm{mmHg})$ & $97,7 \pm 7,7$ \\
\hline Pressão arterial diastólica $(\mathrm{mmHg})$ & $61,3 \pm 8,2$ \\
\hline
\end{tabular}

A tabela 2 apresenta os resultados para o Teste Exato de Fisher. Com a análise, foi possível observar associações entre o nível de psicogênese e a escala de desenvolvimento motor (Fisher=12,887; $p=0,009$ ), idade motora geral (Fisher=18,368; $p=0,01$ ), e quociente motor geral (Fisher $=31,092 ; p=0,042$ ). Todavia, quando associados 0 teste de leitura e essas mesmas variáveis não foram encontrados resultados estatisticamente diferentes (Fisher=3,665; $p=0,18$ para escala de desenvolvimento; Fisher $=6,014 ; p=0,12$ para idade motora geral; e Fisher $=3,068 ; p=0,09$ para quociente motor geral).

Tabela 2 - Teste Exato de Fisher entre as variáveis motoras e cognitivas

\begin{tabular}{c|c|c}
\hline & NP & TL \\
\hline ED & $12,887^{*}$ & 3,665 \\
\hline IMG & $18,368^{*}$ & 6,014 \\
\hline QMG & $31,092^{*}$ & 3,068
\end{tabular}

${ }^{\star} p \leq 0,05$, indicando associação entre as variáveis cognitivas e motoras. NP=nível de psicogênese; TL=teste de leitura; ED=Escala de desenvolvimento motor; IMG=idade motora geral; $\mathrm{QMG}=$ quociente motor geral.

A Correlação de Spearman, por sua vez, revelou associações positivas e significativas entre o nível de psicogênese e as demais variáveis. Ademais, também foram encontradas associações significativas entre 0 teste de leitura e a idade motora geral $(r=0,452 ; p=0,012)$ e o quociente motor geral $(r=0,410 ; p=0,025)$, conforme tabela 3 .

Tabela 3 - Correlação de Spearman entre as variáveis motoras e cognitivas

\begin{tabular}{c|c|c|c|c}
\hline & ED & IMG & QMG & NP \\
\hline ED & - & - & - & - \\
\hline IMG & $0,554^{*}$ & - & - & - \\
\hline QMG & $0,396^{*}$ & $0,736^{*}$ & - & - \\
\hline NP & $0,356^{*}$ & $0,475^{*}$ & $0,372^{*}$ & - \\
\hline TL & 0,192 & $0,452^{*}$ & $0,410^{*}$ & $0,833^{*}$ \\
\hline
\end{tabular}

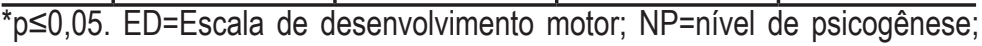

$\mathrm{TL}=$ teste de leitura; IMG=idade motora geral; $\mathrm{QMG}=$ quociente motor geral.

Outros estudos disponíveis na literatura corroboram com os aqui encontrados. Wassenberg et al. ${ }^{11}$, avaliaram crianças de 5 - 6 anos e encontraram uma relação entre 0 desempenho motor e diversas medidas específicas de cognição. Piek et al. ${ }^{12}$, observaram que crianças que desenvolviam suas habilidades motoras mais cedo (até os quatro anos de vida) apresentavam melhor desenvolvimento cognitivo na idade escolar (entre 7 e 11 anos de idade). Murray et al. ${ }^{13}$, constataram uma associação inversa entre o tempo em que a criança demorou a ficar na posição em pé e resultados obtidos em testes cognitivos, quando adulto. Em outras palavras, quanto mais rápido a criança fica em pé, melhores são os resultados dos testes cognitivos quando adulto.

Da mesma forma, estudos têm citado a existência de uma relação entre 0 atraso nos desenvolvimentos motor $e$ cognitivo. Vuijk et al. ${ }^{14}$ relataram que crianças com deficiência intelectual apresentam mais problemas motores do 
que crianças normais. Além disso, os autores citaram uma associação entre o grau da deficiência intelectual e 0 desempenho em testes de destreza manual, habilidades com bola e equilíbrio. Bonifacci et al. ${ }^{15}$ verificaram que crianças com menor habilidade motora possuem uma menor integração óculo-motora. Westendorp et al. ${ }^{16}$ constataram que crianças com baixo QI obtiveram resultados piores em testes motores quando comparados à crianças com QI normal.

Entre as causas do retardo no desenvolvimento cognitivo e motor das crianças estão relacionados o tempo de gestação e o peso ao nascer. Böhm et al. ${ }^{17}$ citaram que crianças nascidas prematuramente possuem mais chances de apresentar uma menor habilidade óculo-motora quando comparadas aos seus pares nascidos a termo. Todavia, a literatura sugere que um programa de intervenção em prematuros pode se mostrar eficaz no auxílio do desenvolvimento cognitivo ${ }^{18}$.

O baixo peso ao nascer também vem sendo abordado nesse sentido. Seitz et al. ${ }^{19}$ relataram que crianças nascidas com menos de $1.250 \mathrm{~g}$ possuem mais chances de terem suas funções motoras e cognitivas prejudicadas quando comparadas a crianças nascidas com peso normal. Park et al. ${ }^{20}$ constataram que indícios de desnutrição precoce possuem influência sobre o desenvolvimento global da criança. Os autores também ressaltaram que um processo de recuperação nutricional tem a capacidade de melhorar o processo de desenvolvimento psicomotor e cognitivo.

Experiências motoras na idade escolar devem reforçar e favorecer as habilidades grafomotoras. Conteúdos da aprendizagem escolar considerados como pré-requisitos da leitura, da escrita e do cálculo devem ser incorporados em atividades lúdicas e motoras, sendo a motricidade fator importante no auxílio de crianças com dificuldades de aprendizagem, uma vez que esse processo depende da interação entre os meios biológico e social, seguindo a hierarquia da maturação (emocional e motora, linguística e cognitiva) ${ }^{21}$. $O$ favorecimento que as experiências motoras exercem na exploração de atividades envolvendo resolução de problemas maximiza a criatividade da criança e seu desejo de explorar novas possibilidades contribuindo, assim, para a aprendizagem escolar da criança ${ }^{22}$.

Nesse contexto, a prática de atividade física pode ser importante para aumentar o acervo motor e cognição das crianças $^{8}$. Verret et al. ${ }^{23}$ relataram que 10 semanas de atividade física melhoraram a capacidade muscular, habilidade motora, comportamento e funções cognitivas em crianças com déficit de atenção e hiperatividade.

Westendorp et al. ${ }^{16}$ constataram que crianças que obtiveram melhores escores em testes com manipulação de objetos participavam mais de práticas esportivas quando comparados aos infantes com baixos escores nesse tipo de teste. Hillman et al. ${ }^{24}$ observaram uma associação direta entre o nível de aptidão física e o desempenho motor em crianças de 9 anos de idade. Em outro estudo, Hillman et al. ${ }^{25}$ relataram que sessões agudas de exercício moderado-intenso $(60 \%$ da frequência cardíaca máxima) podem auxiliar no controle cognitivo da atenção de crianças de 9 anos. Buck et al. ${ }^{26}$ verificaram uma relação entre a aptidão aeróbia e o desempenho no stroop test em crianças de 7 a 12 anos.

No que se refere às associações entre as variáveis antropométricas e os índices motores e cognitivos, não foram observadas associações significativas tanto para o Teste Exato de Fisher como para a Correlação de Spearman (dados não exibidos). Entretanto, alguns estudos disponíveis na literatura relatam o contrário. Mond et al. ${ }^{27}$ verificaram, em crianças alemãs, que meninos obesos tinham habilidades motoras globais prejudicadas quando comparados com meninos eutróficos. Ademais, meninas com excesso de peso possuíam menos habilidade de focar a atenção em relação às meninas com peso normal. Li et al. ${ }^{28}$ observaram uma menor habilidade mental geral em crianças $\mathrm{e}$ adolescentes americanos que estavam acima do peso em relação a seus pares eutróficos. As crianças avaliadas em nosso estudo eram eutróficas e normotensas para o peso, o que pode justificar a falta de associação encontrada.

Possíveis limitações inerentes ao presente estudo se referem à abordagem transversal. Nesse sentido, estudos com um delineamento longitudinal poderiam descrever melhor o comportamento das variáveis estudadas. Além disso, fatores importantes como tempo de gestação e peso ao nascer da criança não foram avaliados. Não obstante, os resultados demonstrados já estabelecem uma associação significativa entre aspectos motores e cognitivos, enfatizando a importância da realização de atividades motoras desde a educação infantil.

\section{Conclusão}

Os resultados do presente estudo revelam uma relação entre desenvolvimento motor e desempenho na leitura e escrita. Foi possível observar que os alunos que obtiveram melhor desempenho nos testes motores também apresentaram escores mais elevados nos testes de leitura e escrita, confirmando a influência do desenvolvimento motor na aprendizagem. Entretanto, não houve associações entre variáveis antropométricas e os índices motores e cognitivos. 


\section{Referências bibliográficas}

1. Meyers R. The twelve Who survive. Strengthening programs of early childhood development in the third world. Michigan: High/Scope, 1995.

2. Meyers R. The twelve Who survive. Londres: Routledge, 1992.

3. Newcombe N. Desenvolvimento infantil - Abordagem de Mussen. $8^{\text {a }}$ Ed. Porto Alegre: Artes Médicas, 1999.

4. Ferreiro E, Teberosky A. Psicogênese da língua escrita. Trad. Diana Myriam Lichtenstein, Liana Di Marco, Mário Corso. Porto Alegre: Artes Médicas Sul, 1999.

5. Le Boulch J. Educação Psicomotora: a psicocinética na Idade Escolar.Porto Alegre: Artmed, 1987.

6. Levin E. A infância em cena. Petrópolis: Ed. Vozes, 2002.

7. MacKay APMG. Atividade verbal: processo de diferença e integração entre fala e escrita. São Paulo: Ed. Plexus, 2001. 8. Pesque C, Crova C, Cereatti L, Casella R, Belucci M. Physical activity and mental performance in preadolescents: effects of acute exercise on free-recall memory. Mental Health and Physical Activity 2009;2:16-22.

9. Rosa Neto F. Manual de avaliação motora. Porto Alegre: Artmed, 2002.

10. Ferreiro E. Psicogênese da língua escrita. Porto Alegre: Artes Médicas, 1999.

11. Wassenberg R, Feron FJM, Kessels AGH, Hendriksen JGM, Kalff AC, Kroes M, et al. Relation between cognitive and motor performance in 5- to 6- year-old children: results from a large-scale cross-sectional study. Child Development 2005;76(5):1092-1103.

12. Piek JP, Dawson L, Smith LM, Gasson N. The role of early fine and gross motor development on later motor and cognitive ability. Human Movement Science 2008;27:668-681.

13. Murray DK, Veijola J, Moilanen K, Miettunen J, Glahn DC, Cannon TD, et al. Infant motor development is associated with adult cognitive categorization in a longitudinal birth cohort study. Journal of Child Psycholody and Psychiatry 2006;41(1):25-29.

14. Vuijk PJ, Hartman E, Scherder E, Visscher C. Motor performance of children with mild intellectual disability and borderline intellectual functioning. Journal of Intellectual Disability Research 2010;54(11):955-965.

15. Bonifacci P. Children with low motor ability have lower visual-motor integration ability but unaffected perceptual skills. Human Movement Science 2004;23:157-168.

16. Westendorp M, Houwen S, Hartman E, Visscher C. Are gross motor skills and sports participation related in children with intellectual disabilities? Research in Developmental Disabilities 2011;32:1147-1153.

17. Böhm D, Lundequist A, Smedler AC. Visual-motor and executive functions in children born preterm: the Bender Visual Motor Gestalt Test revisited. Scandinavian Journal of Psychology 2010;51:376-384.

18. Orton J, Spittle A, Doyle L, Anderson P, Boyd R. Do early intervention programmes improve cognitive and motor outcomes for preterm infants after discharge? A systematic review. Developmental Medicine \& Child Neurology 2009;51:851-859.

19. Seitz J, Jenni OG, Molinari L, Caflisch J, Largo RH, Hajnal DL. Correlations between motor performance and cognitive functions in children born < $1250 \mathrm{~g}$ at school age. Neuropediatrics 2006;37:6-12.

20. Park H, Bothe D, Holsinger E, Kirchner HL, Olness K, Mandalakas A. The impact of nutritional status and londitudinal recovery of motor and cognitive milestones in internationally adopted children. Int. J. Environ. Res. Public Health 2011;8:105-116.

21. Fonseca V. Desenvolvimento Psicomotor e Aprendizagem. Porto Alegre: Artmed, 2008.

22. Gallahue DL, Ozmun JC. Compreendendo o desenvolvimento motor: bebês, crianças, adolescentes e adultos. $2^{\mathrm{a}}$ edição. São Paulo: Phorte, 2003.

23. Verret C, Guay MC, Berthiaume C, Gardiner P, Béliveau L. A physical activity program improves behavior and cognitive functions in children with ADHD: an exploratory study. J of Att. Dis. 2010;XX(X):1-XX.

24. Hillman CH, Buck SM, Themanson JR, Pontifex MB, Castelli DM. Aerobic fitness and cognitive development: eventrelated brain potential and task performance indices of executive control in preadolescent children. Developmental Psychology 2009;45(1):114-129.

25. Hillman CH, Pontifex MB, Raine LB, Castelli DM, Hall EE, Kramer AF. The effect of acute treadmill walking on cognitive control and academic achievement in preadolescent children. Neuroscience 2009;159:1044-1054.

26. Buck SM, Hillman $\mathrm{CH}$, Castelli DM. The relation of aerobic fitness to stroop task performance in preadolescent children. Med. Sci. Sports Exerc. 2008;40(1):166-172. 
27. Mond JM, Stich H, Hay PJ, Kraemer A, Baune BT. Associations between obesity and developmental functioning in pre-school children: a population-based study. International Journal of Obesity 2007;37:1068-1073.

28. Li Y, Dai Q, Jackson JC, Zhang J. Overweight is associated with decreased cognitive functioning among school-age children and adolescents. Obesity 2008;16:1809-1815.

\section{Shirley Holanda da Silva Rocha}

Endereço para correspondência - Rua QS 07, Lote 1, EPCT, Sala G119. Bairro Taguatinga Sul, CEP 72030-170, Brasília, DF, Brasil.

E-mail: shspopgirl@hotmail.com

Currículo Lattes: ---

José Fernando Vila Nova de Moraes - josefernando.moraes@univasf.edu.br

Isabela Almeida Ramos - ahbeuramos@gmail.com

Suliane Beatriz Rauber - suliane.edfisica@gmail.com

Lílian Alves Pereira - lap.edfi@gmail.com

Herbert Gustavo Simões - hgsimoes@gmail.com

Carmen Sílvia Grubert Campbell - campbellcsg@gmail.com

Recebido em 06 de setembro de 2013.

Publicado em 26 de maio de 2014. 
